the behavioral and mood symptoms observed, which were very challenging for caregivers.

\section{THE IMPACT OF LEARNING MULTIPLE NEW SKILLS ON COGNITIVE DEVELOPMENT AND FUNCTIONAL INDEPENDENCE IN OLDER ADULTHOOD}

S. Leanos ${ }^{1}$, E. Kurum², A. Ditta ${ }^{2}$, G. Rebok ${ }^{3}$, R. Wu $\mathrm{Wu}^{1}, 1$. University of California, Riverside, 2. UC Riverside, 3. Department of Mental Health, Johns Hopkins Bloomberg School of Public Health

A new approach, inspired by infant and child development research, suggests that frequently learning a variety of new difficult real-world skills increases cognitive abilities (e.g., working memory and cognitive control) and functional independence (Nguyen et al., under review; Wu et al., 2017). Prior real-world skill learning interventions with older adults typically include only one skill, despite correlational studies demonstrating the benefits of frequent, varied activity engagement (e.g., Carlson et al., 2012). We conducted a 15 -week intervention with older adults engaging in frequent, varied learning of new difficult skills (simultaneously learning Spanish, painting, and how to use tech devices; "intervention group"). A no-contact control group completed only the assessments. The difference in cognitive scores (composite score for working memory and cognitive control) between the intervention and control groups became significant at post-test $(\mathrm{t}=2.36, \mathrm{p}=.03$, effect size $=0.66)$. From pre-test to post-test, the intervention group increased more than one standard deviation in the cognitive scores on average $(\mathrm{M}=.55, \mathrm{SD}=.44$, range .31 to .93$)$. The no-contact control group did not exhibit such changes $(\mathrm{M}=.08, \mathrm{SD}=.43$, range -.83 to .61). The intervention group also increased in their functional independence (Everyday Problems Test) by $12 \%$ on average ( $\mathrm{SD}=12 \%$, range $-1 \%$ to $31 \%)$. The control group declined in their functional independence on average $(\mathrm{M}=-2 \%, \mathrm{SD}=12 \%$, range $-22 \%$ to $16 \%)(\mathrm{t}=2.09, \mathrm{p}=.06)$. Overall, this study demonstrates the potential of an intervention including frequent, varied real-world skill learning in older adults.

\section{THE INTERPLAY BETWEEN DAILY POSITIVE EVENTS AND DAILY STRESS SEVERITY ON INFLAMMATION IN ADULTHOOD}

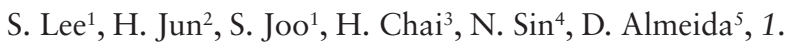
Yonsei University, 2. Yonsei University, Seoul, Republic of Korea, 3. The Pennsylvania State University, 4. University of British Columbia, 5. Pennsylvania State University

In examining the dynamics between positive and negative experiences on health, previous studies suggest two possible pathways - the buffering hypothesis and blunting hypothesis. Buffering hypothesis suggests that positive experiences offset the harmful effects of negative experiences, while blunting hypothesis predicts that negative experiences will nullify the benefits of positive experiences. To test these hypotheses, this study examined interactions between daily positive events and daily stress severity on inflammation and whether the associations differ by age. Sample used were adults aged 34 to $82(\mathrm{~N}=597)$ from the Midlife in the United States Study Wave 2. Reports of respondents' daily experiences of positive events and stress severity were used as predictors, and log-transformed interleukin-6 (IL-6) and
C-reactive protein (CRP) were used as outcome variables. Results showed significant interaction between daily stress severity and daily positive events, with evidence supporting the blunting hypothesis. Specifically, daily positive events were associated with lower levels of IL-6 $(b=-0.16, p<.01)$ and CRP $(\mathrm{b}=-0.27, \mathrm{p}<.01)$ among adults with lower levels of stress severity, but not for those with high stress severity. Buffering hypothesis was not supported; that is, daily positive events did not attenuate the associations between daily stress severity and IL-6 or CRP. Age did not moderate the interaction between daily positive events and stress severity on inflammation. Findings suggest that with severely stressful experiences, the benefits of positive events on inflammation diminishes significantly among midlife and older adults.

\section{THE MODERATING EFFECT OF HEALTH LITERACY ON CHRONIC HEALTH CONDITIONS AND FUNCTIONAL ABILITY}

A. Nehrkorn-Bailey, J. Hicks Patrick, West Virginia

University

Living with chronic health conditions can directly affect physical functioning among younger, middle-aged, and older adults. Although research has focused on the association between health literacy and chronic conditions, the current literature has not assessed whether heath literacy can help to mitigate the effects of chronic conditions on daily functioning among adults. Health literacy, by definition, allows people to have knowledge and understanding of health-related information. Data from a community-based sample of 480 adults (ages 18-85 years; $M=50.2$ ) was utilized to assess the direct association of chronic health condition severity on daily functioning, with age and objective health literacy included in the model as moderators. Hayes' Process macro (Hayes, 2013) was used in SPSS to conduct the analysis. The overall model was significant, $\mathrm{F}(5,372)=34.50$, $\mathrm{p}<$ $.001, \mathrm{R} 2=0.32$, along with a significant main effect for age, $\mathrm{b}=1.41, \mathrm{t}(372)=2.05, \mathrm{p}<.05 ;$ a significant main effect for health literacy, $b=-0.39, \mathrm{t}(372)=-3.04, \mathrm{p}<.01$; and a significant interaction between chronic health conditions and health literacy, $b=0.06, t(372)=3.21, p<.01$. Results are discussed in terms of minimizing the impact of chronic conditions on daily functioning and the importance of health literacy.

\section{THE MORALITY OF LYING TO MY GRANDPARENT WITH DEMENTIA}

A. Ebert ${ }^{1}$, A. Miron ${ }^{2}$, A. Hodel ${ }^{2}$, 1. West Virginia University, 2. University of Wisconsin Oshkosh

We used Gilligan's (1982) morality of care theory to examine how grandchildren cope with having to deceive or lie to their grandparent with dementia. Data from individual semi-structured interviews with thirteen young adults were analyzed using Interpretative Phenomenological Analysis. We found that a moral orientation shift toward a person-centered morality of care occurs gradually. Grandchildren struggle with concepts of moral goodness, importance of close others, and questions about self as a moral agent. We uncovered three antecedents of this shift: (1) severity of dementia symptoms, (2) valuing of the grandparent's welfare, and (3) valuing of the relationship. Further, we discovered this shift has three outcomes: (1) transformation of self as a moral 
agent (experienced emotions become guides to moral behavior, the morality of care orientation affects moral appraisals, behaviors, and emotions within and outside of the grandchild-grandparent relationship), (2) changed relationship with the grandparent, and (3) changed family dynamics and relationships. Current ethics and legislation assume the presence of a common shared world for both people with dementia and their family members (Hertogh et al., 2004). However, when people with dementia no longer share the same reality as their family members, it may be more difficult to use these common ethical standards to guide ethical decisions (Hasselkus, 1997; Hertogh et al., 2004; Moody, 1988; Schermer, 2007). Consequently, work is needed to develop new guidelines for family interactions that take these complex moral issues into consideration.

\section{THE NEGATIVE IMPACT OF UNCONTROLLABLE STRESSORS ON ANXIETY ARE GREATEST FOR PRE- BOOMER GENERATIONS}

\section{Rainville, L. Mehegan, AARP}

This study looks at the relationship between generational status, "uncontrollable" life stressors, and anxiety as measured on the GAD7. We theorize that older generations (i.e. Boomer/ Silents) would have had a greater opportunity to form meaning systems that account for, and make tolerable, stressful life events. To test an implication of this theory, we ran a stressmoderation model using a life stress index constructed of "uncontrollable" stressors for which meaning-making, rather than direct action, is one of few remaining recourses (e.g. grief, personal illness, etc.). Results consistent with an "agingas-coping-resource" theory were observed using data from a probability-based online survey fielded May 15-31, 2018 for AARP among a nationally representative sample of 2,260 adults age 18 and older. Specifically, Pre-Boomer generations were found to have significantly greater anxiety scores than older generations in the high-stressor condition. Subsequent blocks of controls related to life-stage, generational values, and coping styles were entered to explore rival hypotheses. Resulting coefficient values and data visualizations suggest that these measures do not attenuate the original stress-buffering effects in any way. As such, this research is consistent with the view that aging itself, by providing the raw inputs for forming meaning-making systems, serves as a coping resource.

\section{THE PROFILES OF VULNERABILITIES AMONG RETIREES IN U.S.A.}

O. Shin ${ }^{1}$, S. Park ${ }^{2}$, M. Kwak ${ }^{3}$ J. Kang ${ }^{4}$, 1. University of

Illinois at Urbana Champaign, 2. Washington University in Saint Louis, 3. Chinese University of Hong Kong, 4. West Coast Poverty Center

As retirement is widely considered as a major life change in older adults, the extensive research investigated the risk factors of well-being and vulnerability after retirement. As vulnerability is the outcome of complex interactions of multiple risks and lack of resources, the emergence, and evolvement of vulnerability will include multi-dimensional aspects, such as risk to poverty, health, social engagement, and depression. This study aims to explore patterns of multidimensional vulnerability of retirees, and examine the association between profiles of vulnerability and well-being. Our sample included 2,617 retirees who aged $65+$ of Health and Retirement Study. Latent class analysis was utilized to identify the heterogeneous patterns of vulnerability, and then a series of OLS regression analyses were conducted to examine the relationship between patterns of vulnerability and well-being. Four vulnerability patterns were identified: Physical and psychological vulnerable (13\%), Social vulnerable $(35 \%)$, Low vulnerable $(37 \%)$ and Material vulnerable $(15 \%)$. The regression analysis showed that those who are in material vulnerable, social vulnerable, physical and psychological vulnerable groups had significantly lower well-being compared to those who are in low-vulnerable group after controlling for age, gender, education, and retirement voluntariness. In particular, physical and psychological vulnerability predicted the lower well-being than other types of vulnerability. Our study provides the empirical evidence that various patterns of vulnerability predicted different levels of well-being after retirement. This result expanded the current literature on retirement by suggesting the potential risk of the physical and psychological vulnerable group in postretirement. Implications for future research and services will be discussed.

\section{THE RELATIONSHIP BETWEEN RETIREMENT EXPECTATIONS AND JOB INSECURITY AMONG THE AGING US WORKFORCE}

B. Gaines ${ }^{1}$, A. Dugan ${ }^{2}$, M. Cherniack ${ }^{2}$, 1. University of

Massachusetts Boston, 2. UConn Health

Retirement insecurity is a rising trend among both workers and retirees in the US. National evidence indicates that many individuals in the labor force expect to work for pay during retirement, and among those, over $50 \%$ expect this postretirement employment to be a source of income for them. In addition, reports show that current retirees are less likely to expect to be able to meet household expenses and cover health-related costs than in 2017. Although feelings of retirement insecurity are on the rise, there is limited understanding of factors driving these concerns. The purpose of this study was to explore potential determinants of retirement insecurity, specifically by assessing the impact of job insecurity. We utilized data from a longitudinal study of manufacturing workers in the state of Connecticut $(\mathrm{N}=452)$ between 2011 and 2013 to examine the relationship between job insecurity and retirement expectations. Using hierarchical multiple regression analysis, our results found both recent and persistent job insecurity were associated with the expectation to work for pay after retirement, the prediction to be unable to meet household expenses during retirement, and the expectation to delay retirement to keep health insurance benefits, even after controlling for age, gender, income, level of supervisory responsibility, and job type. These findings suggest that developing and implementing strategies for workers to cope with job insecurity may help alleviate retirement-related concerns. Given recent reported improvements in the US economic climate, future examination of this topic involving more recent data is needed to determine if this relationship persists.

\section{THE ROLE OF AGE AND CULTURE IN UNDERSTANDING SOCIAL AND EMOTIONAL PROCESSES IN DAILY LIFE}

C. Arredondo ${ }^{1}$, G. Luong ${ }^{1}$, K. Fingerman ${ }^{2}$, 1. Colorado

State University, 2. University of Texas at Austin 